\title{
APLICABILIDAD DE SIMULADORES DE MEDIANA COMPLEJIDAD EN EL PROCESO DE FORMACIÓN DE RESIDENTES DE GINECOLOGÍA Y OBSTETRICIA
}

\author{
M. Angeles Baeza A. ${ }^{a}$, Javiera Mella B. ${ }^{a}$, Arturo Soldati O. ${ }^{1}$, Eliana Escudero Z. ${ }^{b}$, \\ Augusto Tapia S. ${ }^{2}$, José Antonio Arraztoa V. ${ }^{1}$ \\ 1 Departamento de Ginecología y Obstetricia y Biología de la Reproducción, Facultad de Medicina, Universidad de los \\ Andes, Hospital Parroquial de San Bernardo. ${ }^{2}$ Departamento de Ginecología y Obstetricia, Clínica Dávila.
}

a Alumnas, Escuela de Medicina, Universidad de los Andes. ${ }^{b}$ Enfermera, Escuela de Enfermería, Universidad Finis Terrae.

\section{RESUMEN}

Antecedentes: El entrenamiento de técnicas quirúrgicas durante la formación de gineco-obstetras encuentra dificultades por razones de seguridad de la paciente y del recién nacido. Una de las estrategias utilizadas es el uso de simuladores para facilitar la adquisición de las habilidades necesarias para resolver situaciones críticas frecuentes en la práctica médica, salvaguardando la ética en la enseñanza y mejorando su calidad. Objetivos: Evaluar el uso de simuladores de parto interactivo de mediana complejidad (Noelle® Gaumard Scientific, Miami, FL) como método de educación médica para adquirir las destrezas necesarias en la atención de un parto operatorio con Espátulas de Thierry. Método: Diseño: estudio piloto prospectivo controlado. Sujetos: seis médicos en formación de la especialidad de la Universidad de los Andes. Se definieron aleatoriamente 2 grupos estratificados por año de formación. A todos se les instruyó en sesión teórica sobre el uso de las espátulas. La mitad tuvo una sesión práctica. Se utilizó un simulador y pautas estandarizadas para evaluar la adquisición de habilidades. Resultados: Se expresó según el porcentaje del puntaje máximo logrado para cada prueba. El grupo experimental tuvo un resultado en la evaluación práctica de 90/25/90\%. El grupo control 35/70/65\%. Se observó menos diferencias inter grupos en la prueba teórica. Conclusión: Las habilidades asociadas al uso de las espátulas de Thierry se adquirieron más completamente en el grupo expuesto a un taller con simuladores de mediana complejidad.

\section{PALABRAS CLAVE: Educación médica, espátulas de Thierry, fantoma}

\section{SUMMARY}

Background: The training on surgical techniques during the instruction of obstetrician-gynecologists finds some difficulties because of patient and newborn's safety concern. One of the strategies is to use simulators that facilitate the acquisition of the skills required to solve critical situations, common in medical practice. Objective: To evaluate the use of simulators (Noelle $\mathrm{M}$ Gaumard Scientific, Miami, FL) in medical education as a mean to acquire the skills needed for an instrumental delivery using Thierry's spatulas. Methods: Design: prospective controlled pilot study. Participants: six medical residents of obstetrics and gynecology from Los Andes University. They were randomized on two groups stratified by years of residency. All of them received a lecture on Thierry's spatulas. Half of them also had a workshop. A simulator and standardized guidelines 
were used to evaluate the skills acquisition. Results: They were stated by the percentage of maximum score achieved to each test. The experimental group had a practical score of 90/25/90\%. The control group got $35 / 70 / 65 \%$. There were few differences in the written test between groups. Conclusion: The experimental group showed a better performance in the use of Thierry's spatulas.

\section{KEYWORDS: Medical education, Thierry's spatulas, phantom}

\section{INTRODUCCIÓN}

La evaluación adecuada del desempeño de un estudiante de medicina, tanto de pre como posgrado, incluye métodos que permitan la valoración de la adquisición de conocimientos, habilidades y comportamientos.

Todos los métodos de evaluación tienen ventajas y desventajas, como la dificultad en la estandarización de la puntuación y la subjetividad dada por las diferencias entre los evaluadores (1). Existen actualmente varios métodos, tanto de alta como de baja confiabilidad, que proveen de una evaluación objetiva (2). Algunos de éstos están bien establecidos, mientras otros están aún en proceso de aprobación (3).

La adquisición de las habilidades de los médicos que cursan especialidades quirúrgicas ha sido evaluada a lo largo del tiempo de forma subjetiva, basándose generalmente en el desempeño anual de éstos, lo cual tiene una baja confiabilidad y validez, influenciada por factores que no reflejan necesariamente sus destrezas $(4,5)$.

La enseñanza efectiva de técnicas quirúrgicas es vital para el entrenamiento de gineco-obstetras. Así mismo, es necesario capacitar para el enfrentamiento de una serie de situaciones clínicas potencialmente fatales, como hemorragias masivas, embolias de líquido amniótico, retención de hombros, entre otras. El problema al que se ve enfrentado el estudiante e incluso el especialista novel, es la falta de exposición a estas situaciones críticas, por lo que su entrenamiento es fundamentalmente teórico, lo que no siempre permite adquirir las competencias necesarias (6). Recientemente algunos programas han incluido laboratorios de entrenamiento de distintas técnicas en objetos inanimados (7). El uso de simuladores permite el asesoramiento de competencias quirúrgicas y puede mejorar las habilidades y desempeño en situaciones reales (8).

Los simuladores han encontrado su aplicación en medicina, donde facilitan la adquisición y mantenimiento de las habilidades y conocimientos ne- cesarios en situaciones críticas frecuentes en la práctica médica, salvaguardando la ética en la enseñanza y mejorando su calidad (9).

La atención de partos vaginales operatorios es parte integral de la formación en ginecología y obstetricia. Clásicamente en Chile se ha utilizado el fórceps de Kielland, por lo que la adquisición de la destreza en el uso de otros instrumentos es difícil (10). Es el caso de las espátulas de Thierry, la cual tiene algunas ventajas sobre el fórceps Kielland. Así, tanto en la experiencia nacional como internacional se ha descrito diferencias en los resultados respecto de las lesiones maternas y del recién nacido (10). Varios son los países de Europa y Latinoamérica que ya han reemplazado el fórceps de Kielland por las espátulas rectas y paralelas, que facilitan el desarrollo exitoso de tales partos (10).

De esta manera, y considerando que existen pocos estudios que muestren el uso de simuladores en la formación de estudiantes de posgrado en Chile, se realizó un taller piloto en la Universidad de los Andes para el entrenamiento en el uso de espátulas de Thierry, junto con su evaluación estandarizada. El objetivo primario de esta publicación es dar a conocer esta experiencia y secundariamente, motivar a crear pautas estandarizadas para la educación médica y su consiguiente evaluación. Los objetivos de esta experiencia son:

- Evaluar el uso de simuladores de parto interactivo de mediana complejidad como método de educación médica para lograr destrezas en la atención de un parto operatorio con espátulas de Thierry.

- Comparar su efectividad según el año cursado por el residente.

\section{MATERIAL Y MÉTODO}

Para el diseño de la investigación se realizó un estudio piloto prospectivo controlado. El estudio se aplicó a los residentes de ginecología y obstetricia de primer a tercer año $(n=6)$ de la Universidad de los Andes. Se creó una guía de parto vaginal operatorio con espátulas de Thierry, en la que se 
dispusieron los conocimientos teóricos anatómicos y técnicos necesarios para la realización de esta práctica obstétrica.

Durante el mes de mayo de 2009, en el Hospital Parroquial de San Bernardo, se realizó un curso de dos días de duración, iniciado con evaluación diagnóstica teórica con puntaje máximo de 14, para luego proceder a una exposición audiovisual sobre el uso de las espátulas. Se distribuyó aleatoriamente a un residente de cada año, formando un grupo control y un grupo experimental. Sólo el grupo experimental realizó un taller práctico con simuladores de mediana complejidad. Se utilizó el simulador de parto Noelle ${ }^{\circledR}$ (Gaumard Scientific, Miami, FL) (Figura 1). Se trata de un simulador de parto vaginal de mediana complejidad de tamaño real, que utiliza un sistema mecánico para la simulación del proceso de parto.

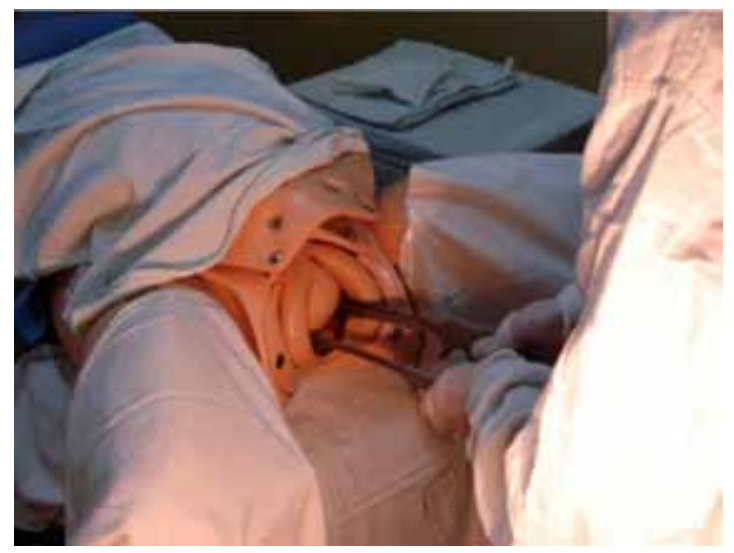

Figura 1. Demostración del uso de las espátulas de Thierry en un simulador de mediana complejidad durante curso práctico.

Ambos grupos fueron controlados posteriormente por un solo evaluador, utilizando el simulador Noelle $\AA$ en un escenario de caso clínico que requería de parto operatorio. Se evaluó según una pauta de cotejo (puntaje máximo: 20) las distintas destrezas definidas (Anexo 1). Posterior a esto, los residentes involucrados realizaron una evaluación escrita sobre las debilidades y fortalezas del curso. Los datos fueron recogidos y expuestos de forma descriptiva, utilizando como medida el porcentaje del puntaje máximo logrado para cada evaluación, a modo de comparar los datos según año de especialidad, realización del curso práctico y conocimientos teóricos de cada uno de los evaluados.

\section{RESULTADOS}

Los resultados obtenidos para las evaluaciones prácticas y teóricas se presentan en la Tabla I. El desempeño en la evaluación teórica es similar entre los residentes, sin observarse una diferencia marcada en relación al año de práctica. La evaluación práctica del grupo control muestra una diferencia dada por los años de formación, la cual se ve francamente atenuada en el grupo experimental (Figura 2).

Con respecto a la evaluación de la actividad, los residentes concuerdan en que es una práctica muy útil en el aprendizaje de la técnica, así como en la seguridad frente a un parto vaginal operatorio. En consecuencia debería realizarse con mayor frecuencia durante los años de formación de ginecoobstetricia. A su vez los residentes coinciden en que la mayor dificultad se presentó al momento de la rotación de las ramas.

\section{DISCUSIÓN}

En los resultados expuestos se puede ver la tendencia a la obtención de un mayor puntaje en aquellos que realizaron el curso práctico previo a la evaluación (grupo experimental) y a medida que es mayor el año cursado de especialidad. Sin embargo, como era esperable, no se puede concluir si es significativo el cambio entre un grupo y otro por el número de participantes. Esta tendencia concuerda con lo publicado en la literatura. En un trabajo realizado por Banks y cols (8), en el uso de simuladores para prácticas gineco-obstétricas, se concluyó que los residentes que habían realizado el laboratorio práctico mejoraban considerablemente su desempeño en la evaluación, así como también en la consolidación de los conocimientos teóricos (8). Macedonia y cols (11), presentó similares resultados en la adquisición de habilidades para parto operatorios por parte de residentes. Otro estudio realizado por Kolkman y cols (12), evalúa distintas destrezas de la práctica gineco-obstétrica según año de residencia, obteniéndose un mejor rendimiento en aquellos cursando los últimos años.

La utilización de simuladores como recurso complementario para la formación médica en general y el entrenamiento de destrezas en particular, aparece cada vez de mayor importancia y utilidad, especialmente para aquellas situaciones clínicas de poca frecuencia pero de alto riesgo (13). La disminución de la tasa de natalidad en nuestro país (14), así como los cambios en las conductas obstétricas que redundan en disminución de partos vaginales, también afectan la adquisición de destrezas operatorias para resolver situaciones que así lo ameriten. 


\section{Anexo I}

PAUTA DE EVALUACION DE APRENDIZAJES Y DESTREZAS EN EL USO DE LAS ESPATULAS DE THIERRY

\begin{tabular}{|c|c|c|c|}
\hline ACCIÓN A EVALUAR & PUNTAJE 2 & PUNTAJE 1 & PUNTAJE 0 \\
\hline Explica el procedimiento & $\begin{array}{l}\text { Saluda y explica el proce- } \\
\text { dimiento a realizar. Pide } \\
\text { consentimiento. }\end{array}$ & $\begin{array}{l}\text { Saluda y explica. No pide } \\
\text { consentimiento. }\end{array}$ & $\begin{array}{l}\text { Solo saluda/solo expli- } \\
\text { ca. }\end{array}$ \\
\hline $\begin{array}{l}\text { Menciona condiciones ade- } \\
\text { cuadas para parto operatorio }\end{array}$ & Nombra los 8 requisitos. & Nombra entre 4 y 8. & Nombra menos de 4. \\
\hline \multicolumn{4}{|l|}{$\begin{array}{l}\text { 1. Dilatación cervical com- } \\
\text { pleta }\end{array}$} \\
\hline \multicolumn{4}{|l|}{ 2. Membranas rotas } \\
\hline \multicolumn{4}{|l|}{ 3. Cabeza encajada } \\
\hline \multicolumn{4}{|l|}{ 4. Feto vivo } \\
\hline \multicolumn{4}{|l|}{$\begin{array}{l}\text { 5. Diagnóstico de la variedad } \\
\text { de posición cefálica }\end{array}$} \\
\hline \multicolumn{4}{|l|}{$\begin{array}{l}\text { 6. Proporcionalidad fetopél- } \\
\text { vica }\end{array}$} \\
\hline \multicolumn{4}{|l|}{ 7. Vejiga vacía } \\
\hline \multicolumn{4}{|l|}{$\begin{array}{l}\text { 8. Anestesia materna satis- } \\
\text { factoria }\end{array}$} \\
\hline Revisa las espátulas & Sí & & No \\
\hline $\begin{array}{l}\text { Introduce la } 1^{\underline{a}} \text { rama (pos- } \\
\text { terior): derecha o izquierda } \\
\text { según la mano guía. }\end{array}$ & Satisfactorio & & Deficiente \\
\hline $\begin{array}{l}\text { Introduce la } 2^{a} \text { rama, con la } \\
\text { mano guía }\end{array}$ & Satisfactorio & & Deficiente \\
\hline Articulación de las ramas & Sí & & No \\
\hline $\begin{array}{l}\text { Verificación de la toma (si- } \\
\text { metría de las espátulas, en } \\
\text { paralelo) }\end{array}$ & Sí & & No \\
\hline $\begin{array}{l}\text { Tracción en sentido del ca- } \\
\text { nal del parto }\end{array}$ & Satisfactorio & & Deficiente \\
\hline $\begin{array}{l}\text { Desprendimiento de polo ce- } \\
\text { fálico y protección del periné }\end{array}$ & Ambas acciones & $\begin{array}{l}\text { Desprendimiento del polo } \\
\text { cefálico sin protección del }\end{array}$ & No logra ninguna \\
\hline Tiempo de la competencia & Menor a 5 minutos & periné & Mayor a 5 minutos \\
\hline
\end{tabular}


Tabla I

PORCENTAJE DEL PUNTAJE TOTAL LOGRADO PARA LA EVALUACIÓN PRÁCTICA Y TEÓRICA, POR AÑO DE RESIDENCIA (R Y NÚMERO) Y SEGÚN GRUPO CASO O CONTROL

\begin{tabular}{lrrrrrr}
\hline & \multicolumn{3}{c}{ Grupo control } & \multicolumn{2}{c}{ Grupo experimental } \\
\hline Evaluación teórica (máx 14) & R1 & 12 & $85,7 \%$ & R1 & 11 & $78,6 \%$ \\
& R2 & 9 & $64,3 \%$ & R2 & 8 & $57,1 \%$ \\
& R3 & 11 & $78,6 \%$ & R3 & 10 & $71,4 \%$ \\
Evaluación práctica (máx 20) & R1 & 7 & $35 \%$ & R1 & 18 & $90 \%$ \\
& R2 & 14 & $70 \%$ & R2 & 5 & $25 \%$ \\
& R3 & 13 & $65 \%$ & R3 & 18 & $90 \%$ \\
\hline
\end{tabular}

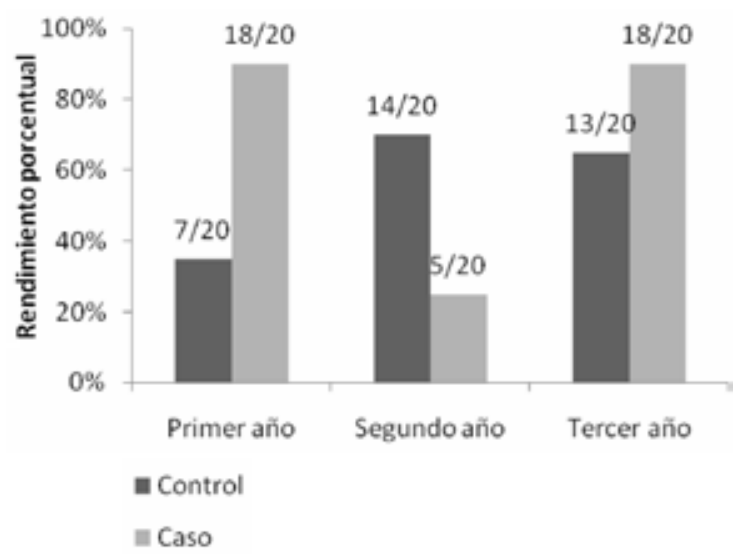

Figura 2. Rendimiento porcentual en la evaluación práctica según caso y control, por año de residencia.

Hay una amplia experiencia en el uso de simuladores en otros campos del saber, siendo su uso para la formación de pilotos de aviones probablemente el más extendido (7). En el campo de la medicina su uso se ha ido difundiendo cada vez más frecuentemente. Los anestesistas tienen gran experiencia al respecto (15), pero el uso en obstetricia y ginecología también ha ido en aumento (16). Las características peculiares de esta especialidad, donde las situaciones críticas exigen una resolución pronta y expedita, y no son de gran frecuencia, hace que la adquisición de las habilidades para enfrentarlas exitosamente pueda ser difícil de lograr. Poca exposición y poco tiempo para enseñar, atentan contra el objetivo de transmitir el conocimiento. El uso de situaciones clínicas simuladas puede, sin duda, ayudar a lograr ese objetivo sin poner en riesgo a la madre e hijo.
El uso de la simulación exige ciertas reglas para que sus objetivos puedan cumplirse. Dependerá del tipo de simuladores que se usen, el grado de realismo que se le quiera dar al ejercicio, pero es necesario tener previamente definido los objetivos a conseguir, una pauta que permita evaluar y una discusión posterior con el educando para mostrar las fortalezas y debilidades mostradas en el ejercicio. Cuando se utilizan escenarios más complejos, como la atención de un parto o el manejo de una hemorragia masiva, es importante definir los roles de los participantes y realmente ponerse en la situación que se quiere recrear. Se puede agregar, además de los simuladores inertes, actores para interactuar. Este juego de roles permite a los formadores destacar aspectos no técnicos, como liderazgo, compañerismo, compasión, empatía, además de los estrictamente técnicos (conocimientos teóricos, destrezas prácticas).

A su vez, estas instancias son evaluadas de manera positiva por parte de los residentes, lo que se observa tanto en la literatura como en nuestra experiencia actual. Según Goff y cols (13), los ejercicios formales en técnicas quirúrgicas proveen una orientación a los residentes de gineco-obstetricia catalogada como "muy útil" por los participantes, ya que les da experiencia, confianza y habilidades básicas previas al enfrentamiento al paciente real.

\section{CONCLUSIÓN}

Creemos que ésta es una iniciativa favorable para el desarrollo del área de educación médica, ya que propone una manera objetiva de evaluación, así como un espacio para que los alumnos puedan desarrollar destrezas y habilidades, enfrentándose con una mayor seguridad al paciente, dado el contexto actual de la práctica médica en pacientes reales. 


\section{BIBLIOGRAFÍA}

1. Casey P, Goepfert A, Espey E, Hammoud M, Kaczmarczyk J, Katz N, et al. To the point: reviews in medical education - the objective structured clinical examination. Am J Obstet Gynecol 2009;200:25-34.

2. Hammoud M, Nuthalapaty F, Goepfert A, Casey P, Emmons S, Espey E, et al. To the point: medical education review of the role of simulators in surgical training. Am J Obstet Gynecol 2008;199:338-43.

3. Darzi A, Datta V, MacKay S. The challenge of objective assessment of surgical skill. Am J Surg 2001;181:484-6.

4. Goff B, Nielsen P, Lentz G, Chow G, Chalmers R, Fenner D, et al. Surgical skills assessment: a blinded examination of obstetrics and gynecology residents. Am J Obstet Gynecol 2002;186:613-7.

5. Morgan P, Cleave-Hogg D. Evaluation of medical students' performance using the anaesthesia simulator. Med Educ 2000;34:42-5.

6. Blum R, Gairing-Bürglin A, Gisin S. Simulation in obstetrics and gynecology - a new method to improve the management of acute obstetric emergencies. Ther Umsch 2008;65:687-92.

7. Macedonia C, Gherman R, Satin A. Simulation laboratories for training in obstetrics and gynecology. Obstet Gynecol 2003;102:388-92.

8. Banks E, Pardanani A, King M, Chudnoff S, Damus $\mathrm{K}$, Comerford M. A surgical skills laboratory improves residents' knowledge and performance of episiotomy repair. Am J Obstet Gynecol 2006;195:1463-7.
9. Rey G, Visconti A, Balager E, Martínez J. Uso de simuladores en ginecología y obstetricia. Experiencia en la enseñanza de pregrado. Educación Médica 2006;9(4B):229-33.

10. Lattus J, Paredes A, Junemann K, Martich A, Contreras $P$, Baeza $N$, et al. Espátulas de Thierry versus fórceps de Kjelland. Rev Chil Obstet Ginecol 2003;68:477-86.

11. Macedonia C, Kopelman J, Sarno A, Satin AJ. Development and validation of a model teaching operative vaginal delivery utilizing an anthropomorphic simulator. Am J Obstet Gynecol 2002;187:5106.

12. Kolkman W, Van de Put MA, Van den Hout WB, Trimbos JB, Jansen FW. Implementation of the laparoscopic simulator in a gynecological residency curriculum. Surg Endosc 2007;21:1363-8.

13. Goff B, Lentz G, Lee D, Mandel L. Formal teaching of surgical skills in an obstetric-gynecologic residency. Obstet Gynecol 1999;93:785-90.

14. Donoso E. Descenso de la natalidad en Chile: un problema país. Rev Chil Obstet Ginecol 2007;72:73-5.

15. Friedman Z, Siddiqui N, Katznelson R, Devito I, Bould MD, Naik V. Clinical impact of epidural anesthesia simulation on short- and long-term learning curve: Highversus low-fidelity model training. Reg Anesth Pain Med 2009;34:229-32.

16. Gardner R, Raemer DB. Simulation in obstetrics and gynecology. Obstet Gynecol Clin North Am 2008;35:97-127. 